\section{Kidney \\ Blood Pressure Research}

\title{
Increased Microalbuminuria Risk in Male Cigarette Smokers: Results from the "Olivetti Heart Study" after 8 Years Follow-Up
}

\author{
Antonio Barbato Lanfranco D'Elia Ludovica Perna Anna Molisso \\ Roberto lacone Pasquale Strazzullo Ferruccio Galletti \\ Department of Clinical Medicine and Surgery "Federico II University" Medical School, Naples, Italy
}

\section{Key Words}

Cigarette smoke $\cdot$ Albuminuria $・$ Renal damage $・$ Cardiovascular risk

\begin{abstract}
Background/Aims: Association between cigarette smoke and albuminuria (UA) was already demonstrated in cross-sectional studies and in selected population samples (i.e diabetic patients). This study aims to evaluate, prospectively, the relationship between cigarette smoke and UA in a male adult population sample, with basal normal kidney function, participating in the Olivetti Heart Study (OHS). Methods: Among 994 participants, examined in both 1994-95 and 2002-04, were selected those resulted in both visits smokers $(n=221)$ and non-smokers $(n=416)$ and with basal normal kidney function (GFR $>60 \mathrm{~mL} / \mathrm{min}$ ) and basal albumin/creatinine ratio (ACR $<30 \mathrm{mg} / \mathrm{g}$ ). Results: At baseline, the prevalence of hypertension was $41 \%$, diabetes affected $6.3 \%$ and obesity $17 \%$ of the whole sample. Smokers showed statistically significant lower levels of systolic (SBP) and diastolic blood pressure (DBP) and BMI $(p<0.001)$ compared to non-smokers. There were not basal differences in UA, GFR and metabolic profile. However, at follow-up examination, smokers showed a statistically significant increase in SBP and DBP $(p<0.05)$, but not in GFR and BMI. Moreover, smokers showed a higher risk compared to non-smokers to be in the higher median levels group of UA (OR: 2.17, C.I.95\%: 1.51-3.13; $p$ $<0.001$ ), even after correction for major confounding factors. Further adjustment for basal antihypertensive and hypoglycemic treatment did not change these patterns of association. Conclusion: In a selected male adult population sample, cigarette smoke was independently associated with the development of higher levels of albuminuria over time.
\end{abstract}




\section{Kidney Blood Pressure Research}

\section{Introduction}

Despite the progress achieved with programmes to reduce cigarette smoking consumption, such as the adoption of the WHO Framework Convention on Tobacco Control (FCTC) in 2003 [1], in 2015, 11.5\% of global deaths, about 6.4 million, were imputable to smoking [2].

Overall, in 2015, cardiovascular disease (CVD) was one of the three leading causes plus cancers and chronic respiratory disease, of smoking-attributable disease burden for both sexes respectively $41 \%, 28 \%$ and $20 \%$ [2].

Among the different mechanisms proposed to explain the harmful effect of cigarette smoking on cardiovascular system, only few studies evaluated the effects on kidney function, and only in selected population groups, such as patients with diabetes mellitus or primary kidney diseases like polycystic kidney disease, glomerulonephritis and lupus nephritis, or, recently, in patients with atherosclerotic renal artery stenosis [3].

Moreover, epidemiologic studies proved that cigarette smoking is associated with an increased risk of chronic kidney disease progression and kidney failure in patients affected by diabetes and hypertension [4-5].

Regarding the mechanisms underlying the relation between cigarette smoking and impaired renal function, an increased rate of transition from microalbuminuria to persistent proteinuria, leading to the progression to end stage renal disease [6] and an impaired vasodilatory responsiveness of intrarenal arteries [7], were demonstrated in diabetic patients. Recently, Ohkuma et al. demonstrated a dose and time dependent relation between cigarette smoking and increase of chronic kidney disease (CKD) incidence and urinary albumin-creatinine ratio, in patients with type 2 diabetes [8].

Furthermore, a recent meta-analysis based on 15 prospective cohorts and including more than 65.000 incident CKD patients, suggests a role of smoking on the incidence and progression of CKD, independently of the underlying disease, but, interestingly, there was no association between smoking and proteinuria [9].

Considering the lack of information about the effect of cigarette smoking, in general population, on the risk to develop a subclinical kidney damage, such as the increase of albuminuria, the aim of this analysis was to evaluate the relationship between cigarette smoking and albuminuria in a adult male population sample with normal basal kidney function after a mean follow-up of about 8 years, participating in the "Olivetti Heart Study".

\section{Materials and Methods}

\section{Study population}

The OHS protocol has been described previously [10-11].

The OHS population derived from the male workforce of the Olivetti factories of Pozzuoli (Naples) and Marcianise (Caserta). One thousand seventy nine subjects were seen at baseline examination between May 1994 and December 1995. Participants with basal glomerular filtration rate (GFR) more than $60 \mathrm{ml} / \mathrm{min}$ and urinary albumin/creatinine ratio less than $30 \mathrm{mg} / \mathrm{g}$ were included in the analysis. In order to investigate the influence of cigarette smoking we selected only participants who were smokers, independently of the number of smoked cigarettes per day, that could have changed during time, and never smokers in both basal and final visit (ex smokers at both baseline and follow-up were excluded). Finally, six hundred thirty-seven participants, with a basal normal kidney function and a complete database for anthropometric measures, renal parameters and information regarding smoking habits at both basal and final examination, conducted between November 2002 and May 2004, after an average time of 7.6 yrs (range: 5 to 10 yrs) were included in the analysis.

The study was approved by the local Ethics Committee and participants gave their informed consent to participate. 


\section{Kidney Blood Pressure Research}

Barbato et al.: Increased Microalbuminuria Risk in Male Cigarette Smokers

Procedures

In both visits, at baseline and follow-up, the participants underwent the following procedures.

They were seen in the morning, in a quiet and comfortable environment, while fasting for at least $13 \mathrm{~h}$. We obtained anthropometric measurements, collected a blood sample, and administered a fixed-sequence questionnaire about the subject's medical history and smoking behaviours.

Body weight and height were measured on a standard beam balance scale. Body weight was measured to the nearest $0.1 \mathrm{~kg}$ and height was measured to the nearest $\mathrm{cm}$.

The body mass index (BMI) was calculated as weight in kilograms divided by the square of the height in meters. The measurements were performed with a flexible, non-extendable plastic tape to the nearest $1.0 \mathrm{~cm}$. Both anthropometric and blood pressure measurements were performed by professional operators who had attended training sessions for standardization of the procedures.

Systolic and diastolic (phase V) BP were taken three times, 2 min apart, with a random zero sphygmomanometer (Gelman Hawksley Ltd, Sussex, UK) after the subject had been sitting upright for at least $10 \mathrm{~min}$. The first reading was discarded, and the average of the second and third reading was used for systolic and diastolic BP. Hypertension was diagnosed if the participants had a systolic

BP of 140 or a diastolic pressure of $90 \mathrm{mmHg}$ or higher, or they reported being on a regular antihypertensive regimen.

A fasting venous blood sample was collected and the blood specimens were immediately centrifuged and stored at $-70^{\circ} \mathrm{C}$ until analysed. Serum glucose was measured by automated methods (Cobas- Mira, Roche, Milan, Italy).

Protocol for the study of renal parameters

Baseline. The day before the examination, participants consumed their evening meal at no later than 19:00 h. On the morning of the study, after having first voided, discarded overnight urine and consuming 400 $\mathrm{ml}$ of water, they produced a fasting timed urine collection. The urine was collected during an average time of $235 \mathrm{~min}$ (range 40-450 minutes). The collection time and volume were recorded, and a specimen was used for the analysis. At the mid-point of the urine collection, a blood sample was obtained by venipuncture with the subject in the seated position and without stasis. Creatinine in serum and urine samples were measured by the picric acid colorimetric method. Albumin (mg)/Creatinine(g) Ratio (ACR) was calculated and a basal ACR lower than $30 \mathrm{mg} / \mathrm{g}$ was used to select participants with normal albuminuria excretion. The Cockcroft-Gault formula was used at baseline to estimate the renal clearance.

Follow-up examination. A 24-h urine collection was obtained. On the day before the visit, participants were instructed to discard the first urine of the morning of the collection and to save all urines voided for the following $24 \mathrm{~h}$. Urine was collected in polypropylene bottles, and delivered to the laboratory for immediate storage of the samples at $-70^{\circ} \mathrm{C}$. Serum and urinary creatinine were assessed by the picric acid colorimetric method (Jaffe') using a Cobas-Mira analyzer. Urinary albumin (UA) concentration was measured by an immunoturbidimetric assay (Horiba ABX Diagnostics, Rome, Italy) using a Cobas-Mira analyser (Roche Instrument Center, Rotkreuz, Switzerland). According to the manufacturer's instructions to measure the 24 $\mathrm{h}$ albumin amount excreted $(\mathrm{mg} / 24 \mathrm{~h})$ and to calculate the ACR: According to ADA ${ }^{12}$, we defined micro and macro albuminuria with an ACR between $30 \mathrm{mg} / \mathrm{g}$ and $300 \mathrm{mg} / \mathrm{g}$ and greater than $300 \mathrm{mg} / \mathrm{g}$ respectively. In addition, we also considered microalbuminuria with an albumin excretion between 30 and $300 \mathrm{mg} / 24 \mathrm{~h}$ and macroalbuminuria more than $300 \mathrm{mg} / 24 \mathrm{~h}$.

\section{Statistical analysis}

Statistical analysis was performed using the Statistical Package for Social Sciences (SPSS-PC version 11; SPSS Inc., Chicago, Illinois, USA). Analysis of variance (ANOVA) was used to assess differences between group means when variables were normally distributed. When the variables distribution deviated significantly from normality, they were normalized by log transformation and log-transformed values were used in the analysis. The Mann-Whitney U test was used to evaluate statistical differences between groups when the variables were not normal distributed. The results were expressed as means and range or $95 \%$ confidence intervals (C.I.) unless otherwise indicated. Two-sided p values $<0.05$ were considered statistically significant. 


\section{Kidney Blood Pressure Research}

Barbato et al.: Increased Microalbuminuria Risk in Male Cigarette Smokers

Table 1. Anthropometric characteristics of study population according to cigarette smoking status. Mean (95\% C.I.); p from ANOVA for normal distributed variables and from a *Mann-Whitney U for not normal distributed variables; SBP: systolic blood pressure, DBP: diastolic blood pressure; GFR: glomerular filtration rate; UA: urinary albuminuria ; ACR: albumin /creatinine ratio

\begin{tabular}{|c|c|c|c|c|c|c|}
\hline \multirow[b]{2}{*}{ Parameter } & \multicolumn{2}{|c|}{ Baseline examination } & \multicolumn{4}{|c|}{ Follow-up examination } \\
\hline & $\begin{array}{c}\text { SMOKERS } \\
(\mathrm{n}=221)\end{array}$ & $\begin{array}{c}\text { NON-SMOKERS } \\
(\mathrm{n}=416)\end{array}$ & $\mathrm{p}$ & $\begin{array}{l}\text { SMOKERS } \\
(n=221)\end{array}$ & $\begin{array}{l}\text { NON-SMOKERS } \\
\quad(n=416)\end{array}$ & $\mathrm{p}$ \\
\hline Age (years)* & 50.4 (49.5 to 51.3$)$ & $51.3(50.6$ to 51.9$)$ & NS & 58.4 (57.6 to 59.2 ) & 59.2 (58.6 to 59.8 ) & NS \\
\hline BMI $\left(\mathrm{kg} / \mathrm{m}^{2}\right)$ & $26.3(25.9$ to 26.7$)$ & 27.4 (27.1to 27.7) & $<0.001$ & $26.6(26.2$ to 27.0$)$ & $27.8(27.5$ to 28.1$)$ & $<0.001$ \\
\hline SBP (mmHg)* & $125.8(123.8$ to 127.8$)$ & 131.3 (129.7 to 132.9 ) & $<0.001$ & $136.3(134.3$ to 138.3$)$ & 138.1 (136.7 to 139.5$)$ & NS \\
\hline DBP (mmHg) & $81.9(80.7$ to 83.1$)$ & 85.7 (84.8 to 86.6$)$ & $<0.001$ & 88.1 (86.9 to 89.3$)$ & $89.9(89.0$ to 90.8$)$ & NS \\
\hline Pulse pressure (mmHg) & 43.8 (42.6 to 45.2 ) & $45.6(44.5$ to 46.6$)$ & 0.05 & 48.2 (46.8 to 49.5$)$ & $48.2(47.2$ to 49.2$)$ & NS \\
\hline Heart rate $(\mathrm{bpm})^{*}$ & 60.5 (59.2 to 61.8$)$ & $62.9(62.0$ to 63.8$)$ & 0.001 & $66.4(65.2$ to 67.6$)$ & $68.2(67.3$ to 69.0$)$ & $<0.05$ \\
\hline Serum glucose $(\mathrm{mg} / \mathrm{dl})^{*}$ & 99.7 (96.1 to 103.2 ) & $102.8(100.3$ to 105.1$)$ & $<0.001$ & 101.7 (97.9 to 105.6$)$ & $103.0(100.4$ to 105.6$)$ & NS \\
\hline Serum creatinine $(\mathrm{mg} / \mathrm{dl})^{*}$ & $1.03(1.02$ to 1.04$)$ & $1.04(1.03$ to 1.05$)$ & NS & 1.14 (1.12 to 1.15$)$ & 1.15 (1.14 to 1.16$)$ & NS \\
\hline Temporized urine volume $(\mathrm{ml})$ & 389.9 (364.3 to 415.5$)$ & 436.9 (416.7 to 457.1$)$ & 0.006 & // & // & \\
\hline $24 \mathrm{~h}$ Urine volume (ml) & // & // & & 1468 ( 1405 to 1531$)$ & 1587 (1538 to 1635 ) & 0.004 \\
\hline GFR $(\mathrm{ml} / \mathrm{min})^{*}$ & 90.0 (87.7 to 92.3$)$ & $91.8(90.1$ to 93.5$)$ & NS & $75.0(73.0$ to 77.0$)$ & $76.0(74.6$ to 77.4$)$ & NS \\
\hline ACR $(\mathrm{mg} / \mathrm{g})^{*}$ & 4.5 (3.8 to 5.3 ) & $5.0(4.4$ to 5.6$)$ & NS & 13.7 (8.8 to 18.7 ) & $11.6(8.3$ to 15.0$)$ & $<0.005$ \\
\hline UA $(\mathrm{mg} / 24 \mathrm{~h})^{*}$ & $/ /$ & $/ /$ & $/ /$ & $20.0(13.0$ to 27.1$)$ & 16.3 (11.9 to 20.7$)$ & $<0.005$ \\
\hline
\end{tabular}

Differences in the prevalence of categorical cardiometabolic risk factors between smokers and nonsmokers participants were tested by Chi-square.

Binary logistic regression analysis, using as dependent variable to be in the upper or lower median UA or ACR levels group at follow-up and basal selected anthropometric and biochemical variables as independent factors, was made to identify the potential predictors of UA and ACR at follow-up. To allow a comparative evaluation of the effects of the different factors on the risk to be in the higher median of UA or ACR group, Z scores were calculated for each factor and used for the analysis. Nagelkerke R square was used to estimate the percent of variance in the dependent variable explained by the independent factors. The goodness-of-fit of the final model was evaluated by the Hosmer-Lemeshow test.

\section{Results}

At baseline, mean age of 637 participants included in the analysis was 51.0 years (range 26-74), of them the 35\% ( $n=221$ ) resulted current smokers (CS) whereas the $65 \%$ $(\mathrm{n}=416)$ were never-smokers (NS) at both baseline and follow-up. At first examination, there were not statistically significant age difference between CS and NS. Systolic, diastolic and pulse pressure were lower $(\mathrm{p}<0.05)$ in CS compared to NS as the BMI, while there were not differences in GFR and ACR. CS showed at both examinations a statistically significant lower urinary volume compared to NS. Detailed characteristics of the study population at both baseline and follow-up examination according to smoke behaviours were reported in Table 1. 


\section{Kidney Blood Pressure Research}

Barbato et al.: Increased Microalbuminuria Risk in Male Cigarette Smokers

Table 3. Differences at baseline in selected variables according to final $24 \mathrm{~h}$ urinary albuminuria median and albumin/creatinine ratio median. Mean (95\% C.I.); $p$ from ANOVA for normal distributed variables and from a *Mann-Whitney U for not normal distributed variables; SBP: systolic blood pressure, DBP: diastolic blood pressure; GFR: glomerular filtration rate; UA: urinary albuminuria; ACR: albumin/creatinine ratio

\begin{tabular}{|c|c|c|c|c|c|c|}
\hline Parameter & Low UA $(n=318)$ & High UA (n=319) & $\mathrm{p}$ & Low ACR $(n=318)$ & High ACR $(n=319)$ & $\mathrm{p}$ \\
\hline Age (years)* & $50.3(49.5$ to 51.0$)$ & $51.7(50.9$ to 52.4$)$ & $<0.05$ & $49.9(49.1$ to 50.6$)$ & 52.1 (51.4 to 52.8$)$ & $<0.001$ \\
\hline BMI $\left(\mathrm{kg} / \mathrm{m}^{2}\right)$ & 26.4 (26.1 to 26.7$)$ & 27.6 (27.3 to 28.0$)$ & $<0.001$ & 26.6 (26.3 to 26.9 ) & 27.4 (27.0 to 27.8$)$ & 0.001 \\
\hline SBP $(\mathrm{mmHg}) *$ & $127.0(125.3$ to 128.8 & $131.8(130.0$ to 133.6 & $<0.0011$ & $27.0(125.3$ to 128.7 & $131.8(129.9$ to 133.7$)$ & 0.001 \\
\hline $\mathrm{DBP}(\mathrm{mmHg})$ & $83.3(82.2$ to 84.3$)$ & $85.5(84.5$ to 86.6$)$ & 0.003 & $83.2(82.2$ to 84.2$)$ & 85.7 ( 84.6 to 86.7 ) & 0.001 \\
\hline Pulse pressure (mmHg) & 43.7 (42.6 to 44.8$)$ & $46.2(45.1$ to 47.4$)$ & 0.002 & 43.8 (42.8 to 44.9$)$ & 46.1 (44.9 to 47.3 ) & 0.005 \\
\hline Heart rate $(\mathrm{bpm})^{*}$ & $61.8(60.8$ to 62.8$)$ & $62.4(61.3$ to 63.4$)$ & NS & $61.8(60.8$ to 62.8$)$ & $62.4(61.3$ to 63.4$)$ & NS \\
\hline Serum glucose $(\mathrm{mg} / \mathrm{dl})^{*}$ & 97.9 (96.3 to 99.5$)$ & $105.4(101.8$ to 109.1$)$ & $<0.05$ & 97.8 (96.4 to 99.3$)$ & 105.5 (101.8 to 109.2 ) & $<0.05$ \\
\hline Serum creatinine $(\mathrm{mg} / \mathrm{dl})^{*}$ & $1.04(1.03$ to 1.05$)$ & $1.04(1.02$ to 1.05$)$ & NS & $1.04(1.03$ to 1.05$)$ & $1.03(1.02$ to 1.04$)$ & NS \\
\hline Temporized Urine volume (ml) & 431 (408 to 453$)$ & $410(387$ to 432$)$ & NS & $438(415$ to 461$)$ & $403(381$ to 425$)$ & $<0.05$ \\
\hline $\mathrm{GFR}(\mathrm{ml} / \mathrm{min})^{*}$ & $89.8(87.9$ to 91.8$)$ & 92.5 (90.6 to 94.5$)$ & 0.023 & 91.3 (89.3 to 93.3$)$ & 91.1 (89.1 to 93.0$)$ & NS \\
\hline ACR $(\mathrm{mg} / \mathrm{g})^{*}$ & $3.6(3.1$ to 4.1$)$ & $6.1(5.4$ to 6.9$)$ & $<0.001$ & $3.5(3.0$ to 4.0$)$ & $6.2(5.5$ to 7.0$)$ & $<0.001$ \\
\hline
\end{tabular}

The prevalence of hypertension and obesity between CS and NS were respectively $29 \%$ vs $47 \%$ $(\mathrm{p}<0.001)$ and $12 \%$ vs $19 \%$ $(\mathrm{p}<0.001)$.

At follow-up examination, ACR was significantly increased in comparison with baseline of $7.5 \mathrm{mg} / \mathrm{g}(95 \% \mathrm{CI}$ : 4.8 to $10.2 \mathrm{mg} / \mathrm{g} ; \mathrm{p}<0.001$ ) and 44 subjects (7\%) were classified in the range of microalbuminuria. Both ACR and UA resulted higher in CS $(\mathrm{p}<0.005)$ compared to NS. GFR and systolic blood pressure did not show any statistically significant difference between CS and NS. However as shown in Table 2, CS showed a statistically significant increase of both systolic and diastolic blood pressure levels during time compared to NS $(\mathrm{p}<0.05)$. Otherwise, pulse pressure, BMI and GFR did not significantly change according to smoke habits. The prevalence of diabetes raised from $6.3 \%$ to $11.6 \%$ in the whole population during the followup time, but we did not find any statistically significant difference in the prevalence of diabetes between CS and NS at baseline and follow-up examination.

In order to study the determinants of the urinary albumin excretion in this sample of
Table 4. Differences at follow-up in selected variables according to final 24 hours urinary albuminuria median. Mean (95\% C.I.); $p$ from ANOVA for normal distributed variables and from a *MannWhitney U for not normal distributed variables SBP: systolic blood pressure, DBP: diastolic blood pressure; GFR: glomerular filtration rate; UA: urinary albuminuria; ACR: albumin /creatinine ratio

\begin{tabular}{lccc}
\hline Parameter & Low UA (n=318) & High UA (n=319) & p \\
\hline Age (years)* & $58.2(57.5$ to 58.9$)$ & $59.6(58.9$ to 60.3$)$ & $<0.05$ \\
BMI $\left(\mathrm{kg} / \mathrm{m}^{2}\right)$ & $26.8(26.4$ to 27.1$)$ & $28.0(27.6$ to 28.4$)$ & $<0.001$ \\
SBP (mmHg)* & $133.5(132.1$ to 135.0$)$ & $141.4(139.7$ to 143.1$)$ & $<0.001$ \\
DBP (mmHg)* & $87.5(86.6$ to 88.5$)$ & $91.0(90.0$ to 92.1$)$ & $<0.001$ \\
Pulse pressure (mmHg) & $46.0(45.0$ to 47.0$)$ & $50.3(49.1$ to 51.6$)$ & $<0.001$ \\
Heart rate (bpm)* & $67.1(66.1$ to 68.1$)$ & $68.0(67.0$ to 69.0$)$ & NS \\
Serum glucose $(\mathrm{mg} / \mathrm{dl}) *$ & $98.7(96.3$ to 101.1$)$ & $106.5(102.9$ to 110.0$)$ & $<0.05$ \\
Serum creatinine $(\mathrm{mg} / \mathrm{dl})^{*}$ & $1.14(1.13$ to 1.15$)$ & $1.15(1.13$ to 1.16$)$ & $\mathrm{NS}$ \\
24 h Urine volume (ml) & $1495(1436$ to 1552$)$ & $1595(1543$ to 1649$)$ & 0.011 \\
GFR (ml/min)* & $75.0(73.4$ to 76.6$)$ & $76.3(74.7$ to 77.9$)$ & NS \\
ACR (mg/g)* & $2.6(2.5$ to 2.8$)$ & $22.1(16.7$ to 27.4$)$ & $<0.001$ \\
\hline
\end{tabular}

Table 5. Differences in variables at follow-up according to final albumin /creatinine ratio median. Mean (95\% C.I.); $p$ from ANOVA for normal distributed variables and from a *Mann-Whitney U for not normal distributed variables; SBP: systolic blood pressure, DBP: diastolic blood pressure; GFR: glomerular filtration rate; UA: urinary albuminuria; ACR: albumin / creatinine ratio

\begin{tabular}{lccc}
\hline Parameter & Low ACR (n=318) & High ACR (n=319) & p \\
\hline Age (years)* & $57.8(57.1$ to 58.6$)$ & $60.0(59.3$ to 60.7$)$ & $<0.001$ \\
BMI $\left(\mathrm{kg} / \mathrm{m}^{2}\right)$ & $27.1(26.7$ to 27.4$)$ & $27.7(27.3$ to 28.1$)$ & $<0.05$ \\
SBP (mmHg)* & $134.2(132.7$ to 135.6$)$ & $140.7(139.0$ to 142.5$)$ & $<0.001$ \\
DBP (mmHg)* & $88.1(87.2$ to 89.0$)$ & $90.4(89.3$ to 91.5$)$ & 0.005 \\
Pulse pressure (mmHg) & $46.0(45.0$ to 47.0$)$ & $50.3(49.1$ to 51.5$)$ & $<0.001$ \\
Heart rate (bpm)* & $67.4(66.4$ to 68.3$)$ & $67.7(66.7$ to 68.8$)$ & NS \\
Serum glucose (mg/dl)* & $98.7(96.3$ to 101.0$)$ & $106.5(102.9$ to 110.0$)$ & 0.006 \\
Serum creatinine $(\mathrm{mg} / \mathrm{dl})^{*}$ & $1.15(1.13$ to 1.16$)$ & $1.14(1.13$ to 1.16$)$ & NS \\
24 h Urine volume (ml) & $1552(1497$ to 1607$)$ & $1539(1485$ to 1594$)$ & NS \\
GFR (ml/min)* & $76.6(74.9$ to 78.2$)$ & $74.8(73.2$ to 76.3$)$ & NS \\
ACR (mg/g)* & $2.5(2.4$ to 2.7$)$ & $22.2(16.9$ to 27.5$)$ & $<0.001$ \\
\hline
\end{tabular}




\section{Kidney Blood Pressure Research}

Table 6. Final prevalence of hypertension, diabetes and obesity according to Urinary albuminuria (AU) and Albumin excretion ratio (ACR) median group at follow-up examination

\begin{tabular}{lcccccc}
\hline Diagnosis & Low UA $(\mathrm{n}=318)$ High UA $(\mathrm{n}=319)$ & $\mathrm{p}$ & Low ACR $(\mathrm{n}=318)$ & High ACR $(\mathrm{n}=319)$ & $\mathrm{p}$ \\
\hline Hypertension & $59.1 \%(\mathrm{n}=188)$ & $74.9 \%(\mathrm{n}=239)<0.001$ & $59.4 \%(\mathrm{n}=189)$ & $74.6 \%(\mathrm{n}=238)$ & $<0.001$ \\
Diabetes & $6.3 \%(\mathrm{n}=20)$ & $16.9 \%(\mathrm{n}=53)<0.001$ & $6.4 \%(\mathrm{n}=20)$ & $16.9 \%(\mathrm{n}=53)$ & $<0.001$ \\
Obesity & $15.4 \%(\mathrm{n}=49)$ & $26 \%(\mathrm{n}=83)<0.001$ & $18.2 \%(\mathrm{n}=58)$ & $23.2 \%(\mathrm{n}=74)$ & NS \\
\hline
\end{tabular}

population, participants were divided in two subgroups according to median value of $24 \mathrm{~h}$ UA and of ACR at follow-up. Table 3 shows the baseline characteristics according to the final median group of UA and ACR. In both analysis, participants who resulted at follow-up examination in the higher UA or ACR groups were, at baseline, older, with higher BMI, systolic, diastolic and pulse blood pressure, serum glucose and ACR $(\mathrm{p}<0.05)$. In addition, also the prevalence of hypertension, diabetes and obesity was statistically higher among the participants in the upper median UA and ACR groups at follow-up. These relations were also confirmed at follow-up examination (see Table 4-6).

Fig. 1 shows the percentage of participants in the upper median levels of UA and ACR according to smoke status. The prevalence of CS in the upper median albuminuria and ACR levels group was higher than NS $(\mathrm{p}<0.05)$. Finally, binary logistic regression analysis, using ACR (high vs low median) at follow-up as dependent variable, shows that the risk to be in the upper median ACR group in CS was statistically higher than in NS (OR: 2.10, 95\%CI: 1.45 to $3.04 ; \mathrm{p}<0.001$ ). Also including in the model the follow covariates: age, systolic blood pressure, serum glucose, temporized

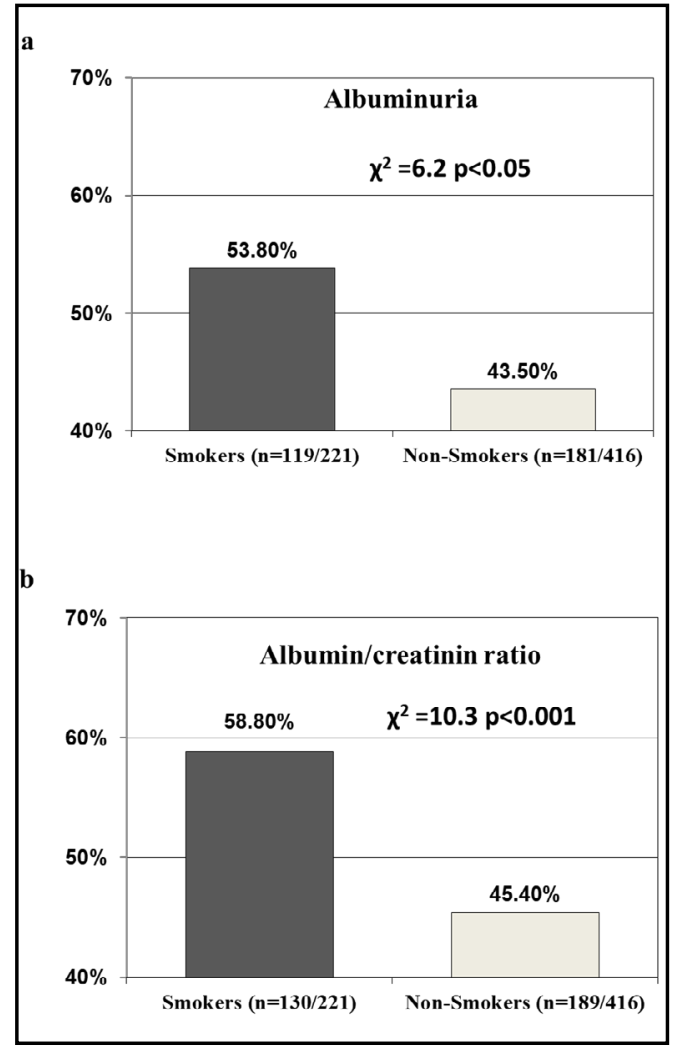

Fig. 1. Percentage of participants in the upper median levels of UA at follow-up examination according to smoke status urine volume and basal ACR, (Table 7). A similar result was found using the same model with UA (high vs low median) at follow-up as dependent variable (OR: 2.28, 95\% CI: 1.57 to 3.29; p<0.001) (Table 7). The adjustment for basal antihypertensive and hypoglycemic treatment did not change these patterns of association between smoke and UA or ACR. Both models explained about 18\% (Nagelkerke $\mathrm{R}$ Square $=0.183$ ) of the overall risk to follow in the high ACR or UA median group at followup.

\section{Discussion}

This is the first study in which the influence of smoking on renal function was investigated in subjects with normal renal function. In this sample of Italian adult male population, after 8 years of follow-up, CS show a greater risk to develop higher level of both $24 \mathrm{~h} \mathrm{UA}$ excretion and ACR, compared to NS. This relation is independent from other well-known 


\section{Kidney Blood Pressure Research}

Table 7. Binary logistic regression analysis considering the risk to be in upper median level of ACR or albuminuria as dependent variable and z-score of age, SBP, glucose, BMI and ACR at baseline as dependent factors $(\mathrm{n}=637)$

\begin{tabular}{|c|c|c|c|c|c|}
\hline Value & OR & $95 \%$ IC & $\mathrm{p}$ & Adjusted R Square & Hosmer-Lemeshow Test \\
\hline & \multicolumn{5}{|c|}{ Dependent variable: ACR (risk to be in the upper median levels of ACR) } \\
\hline Smoke (yes/no) & 2.10 & $1.45-3.04$ & $<0.001$ & & \\
\hline ACR (z-score) & 1.69 & $1.38-2.07$ & $<0.001$ & & \\
\hline BMI (z-score) & 1.55 & $1.29-1.87$ & $<0.001$ & & \\
\hline Serum glucose (z-score) & 1.33 & $1.07-1.67$ & 0.011 & 0.181 & 0.840 \\
\hline Systolic Blood Pressure (z-score) & 1.10 & $0.91-1.34$ & NS & & \\
\hline Age (z-score) & 1.08 & $0.90-1.30$ & NS & & \\
\hline \multirow[t]{2}{*}{ Temporized Urine volume (z-score) } & 0.80 & $0.67-0.95$ & 0.014 & & \\
\hline & \multicolumn{5}{|c|}{ Dependent variable: Albuminuria (risk to be in the upper median levels of albuminuria) } \\
\hline Smoke (yes/no) & 2.28 & 1.57-3.29 & $<0.001$ & & \\
\hline ACR (z-score) & 1.73 & $1.41-2.13$ & $<0.001$ & & \\
\hline Serum glucose (z-score) & 1.38 & $1.09-1.76$ & 0.007 & & \\
\hline BMI (z-score) & 1.29 & $1.07-1.54$ & 0.006 & 0.183 & 0.676 \\
\hline Age (z-score) & 1.26 & $1.05-1.52$ & 0.013 & & \\
\hline Systolic Blood Pressure (z-score) & 1.09 & $0.90-1.32$ & NS & & \\
\hline Temporized Urine volume (z-score) & 0.79 & $0.66-0.94$ & 0.009 & & \\
\hline
\end{tabular}

risk factors such as hypertension, diabetes and obesity and in agreement with the previous demonstration, in the same study population, that smoking cessation was associated with lower blood pressure increment and minor hypertension risk [13].

This relation was so far evaluated only in selected groups of population, such as patients affected by diabetes mellitus, primary kidney disease [3] or hypertension [4]. Our data indicated, for the first time, that in subjects without evidence of initial renal damage, i.e. with an ACR $<30$, the amount of albumin lost during the time in the urine is smoking dependent. It is important to note that the groups with higher ACR or albuminuria at follow-up have a cardiovascular risk profile significantly higher than subjects with values below the median.

In agreement with our results, many studies showed that the relationship between albuminuria and adverse outcomes usually becomes evident for increasing albuminuria even in the limit of normoalbuminuria [14]. However, albuminuria is also a risk factor for CVD in people with and without diabetes [15]. Among possible mechanisms, an early smokingassociated glomerular hyperfiltration [8], due to alterations in kidney hemodynamics, blood pressure and impairment of kidney microvasculature [16], associated to a subsequently renal dysfunction was hypothesized.

In particular, it was proposed that the effects of cigarette smoking on kidney were linked to the extension of atherosclerosis to renal microvasculature [17], through a vascular endothelial cell damage due to the increase in oxidative stress and activation of leukocytes, that results in increased release of inflammatory cytokines, promoting the action of matrix metalloproteinase, activating platelet aggregation, increasing plasma fibrinogen, augmenting clot strength, and reducing fibrinolytic capacity [18].

Another proposed link between cigarette smoking and renal damage is cadmium, found four times higher in blood of smokers compared to non-smokers [19].

Cadmium is an environmental pollutant absorbed into the body, even if not exclusively, by cigarette smoking, with a slow excretion rate due to renal reabsorption [20]. Among the pathologic effects, chronic low level exposures to cadmium, that exhibit extensive half-life (30 years), can cause nephrotoxicity leading to proteinuria, calciuria, glycosuria, and tubular necrosis [19].

Among the effect of smoke on renal hemodynamics, a significant increase of mean arterial pressure accompanied by a significant increase in arginine vasopressin (VP) was previously reported in healthy and nephropathic patients [21]. In particular, VP is secreted by pituitary gland as a response to increased serum osmolality and V2 receptor mediated action was associated to development of albuminuria in rats and human [22-23]. 


\section{Kidney Blood Pressure Research}

Cigarette smoking could increase through nicotine-mediated mechanisms the release of VP by stimulation of central cholinergic projections to hypothalamus [24]. It was demonstrated, however, that low-rate nicotine infusion, not able to induce nausea, vomiting or decrease in blood pressure, does not induce vasopressin release, as instead happens with smoking, suggesting an airway-related mechanism [25]. Furthermore, elevated serum copeptin, the C-terminal fragment of the vasopressin pro-hormone, is linked to an increased risk of developing albuminuria, independently of incident diabetes and hypertension [26]; current smoking is associated with higher serum copeptin [27]. According to the VP hypothesis, our data confirmed that CS showed a significantly lower urinary volume compared to NS at baseline and follow-up (see Table 1).

The limitation of this study are largely due to its nature of epidemiological investigation and our results are generalizable only to a comparable white adult male population.

Several studies showed that the risk of renal impairment associated with smoking was higher in men compared to women despite the different origin of study population (patients with primary renal disease or type 1 diabetic nephropathy) [28]. Then, the lack of women could have influenced our results; however the findings of this analysis, also if not generalizable to the whole population, are certainly interesting as regards the male population.

At baseline, the lack of a $24 \mathrm{~h}$ urine collection led to the use of a surrogate formula such as the Cockcroft-Gault (CG) formula to define the population with normal renal function. However, CG formula is considered of interest in screening declining renal function in subjects with normal serum creatinine (SC) in order to investigate sample of population at risk [29]. Moreover, CG predicts mortality better than the CKD-EPI and MDRD in a recent study aiming to assess 1-year mortality prediction in patients with heart failure and concomitant atrial fibrillation [30].

Some concerns about this analysis could be due to the use of median to discriminate patients with higher albuminuria levels at follow-up and not of the range of values generally used to define pathological albuminuria (such as those proposed by ADA [12]), but we aim to demonstrate, in a population with basal normal kidney function and without microalbuminuria, the role of cigarette smoking in development of a preclinical renal damage. Furthermore, also if cut-off identified by ACR or albuminuria median falls in the normal range, the groups in the upper median part were able to identify participants with a statistically significant higher prevalence of cardiovascular risk factors. In agreement with our results, many studies showed that the relationship between albuminuria and adverse outcomes usually becomes evident for increasing albuminuria even in the limit of normoalbuminuria [14]. However, albuminuria is also a risk factor for CVD in both people with and without diabetes [15]. Despite the adjustment for all the others well known cardiovascular risk factors, smoke remains an independent predictor of ACR and UA.

\section{Conclusion}

This analysis shows that in a selected sample of male adult population with normal kidney function and without microalbuminuria, cigarettes smoking was independently associated with the development of higher albuminuria levels during time.

\section{Acknowledgements}

Statement of Ethics: Subjects have given their written informed consent. The study was approved by the local Ethics Committee.

Authors' Contributions: $\mathrm{AB}$ and FG contributed to the analysis design, data interpretation and critical revision, all authors provided input into data analysis. AB and FG wrote the first 


\section{Kidney \\ Blood Pressure Research}

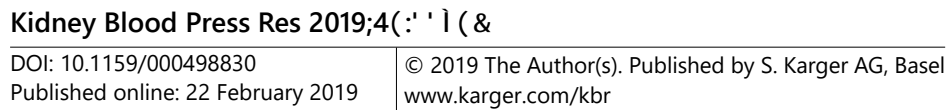

Barbato et al.: Increased Microalbuminuria Risk in Male Cigarette Smokers

draft of manuscript. RI performed the blood and urine assays and contributed to critical revision. LDE contributed to data collection and critical revision. LP, AM and PS contributed data interpretation and critical revision of the manuscript.

\section{Disclosure Statement}

The authors have no conflicts of interest to declare.

\section{References}

-1 Shibuya K, Ciecierski C, Guindon E, Bettcher DW, Evans DB, Murray CJ: WHO Framework Convention on Tobacco Control. WHO Framework Convention on Tobacco Control: development of an evidence based global public health treaty. BMJ 2003;327:154-157.

-2 GBD 2015 Tobacco Collaborators: Smoking prevalence and attributable disease burden in 195 countries and territories, 1990-2015: a systematic analysis from the Global Burden of Disease Study 2015

Lancet 2017;389:1885-1906.

- Drummond CA, Brewster PS, He W, Ren K, Xie Y, Tuttle KR, Haller ST, Jamerson K, Dworkin LD, Cutlip DE, Murphy TP, D’Agostino RB Sr, Henrich WL, Tian J, Shapiro JI, Cooper CJ: Cigarette smoking and cardio-renal events in patients with atherosclerotic renal artery stenosis. PLoS One 2017;12:e0173562.

4 Altay S, Onat A, Özpamuk-Karadeniz F, Karadeniz Y, Kemaloğlu-Öz T, Can G: Renal "hyperfiltrators” are at elevated risk of death and chronic diseases. BMC Nephrol 2014;15:160.

5 Yamagata K, Ishida K, Sairenchi T, Takahashi H, Ohba S, Shiigai T, Narita M, Koyama A: Risk factors for chronic kidney disease in a community-based population: a 10-year follow-up study. Kidney Int 2007;71:159-166.

6 Bentata Y, Karimi I, Benabdellah N, Alaoui FE, Haddiya I, Abouqal R: Does smoking increase the risk of progression of nephropathy and/or cardiovascular disease in type 2 diabetic patients with albuminuria and those without albuminuria? Am J Cardiovasc Dis 2016;6:66-69.

7 Matsumoto N, Ishimura E, Taniwaki H, Emoto M, Shoji T, Kawagishi T, Inaba M, Nishizawa Y: Smoking and proteinuria impair vasodilatory response of intrarenal arteries to nitroglycerine in patients with type 2 diabetes mellitus. Nephrol Dial Transplant 2002;17:608-613.

-8 Ohkuma T, Nakamura U, Iwase M, Ide H, Fujii H, Jodai T, Kaizu S, Kikuchi Y, Idewaki Y, Sumi A, Hirakawa Y, Kitazono T: Effects of smoking and its cessation on creatinine- and cystatin C-based estimated glomerular filtration rates and albuminuria in male patients with type 2 diabetes mellitus: the Fukuoka Diabetes Registry. Hypertens Res 2016;39:744-751.

-9 Xia J, Wang L, Ma Z, Zhong L, Wang Y, Gao Y, He L, Su X: Cigarette smoking and chronic kidney disease in general population: a systematic review and meta-analysis of prospective cohort studies. Nephrol Dial Transplant 2017;32:475-487.

10 Cappuccio FP, Strazzullo P, Farinaro E, Trevisan M: Uric acid metabolism and tubular sodium handling: results from a population based study. JAMA 1993;270:354-359.

-11 Galletti F, Barbato A, Versiero M, Iacone R, Russo O, Barba G, Siani A, Cappuccio FP, Farinaro E, della Valle E, Strazzullo P: Circulating leptin levels predict the development of metabolic syndrome in middle-aged men: an 8-year follow-up study. J Hypertens 2007;25:1671-1677.

12 American Diabetes Association: Standards of medical care in diabetes. Diabetes Care 2005;28:990.

-13 D’Elia L, De Palma D, Rossi G, Strazzullo V, Russo O, Iacone R, Fazio V, Strazzullo P, Galletti F: Not smoking is associated with lower risk of hypertension: results of the Olivetti Heart Study. Eur J Public Health 2014;24:226-230.

14 MacIsaac RJ, Ekinci EI, Jerums G: 'Progressive diabetic nephropathy. How useful is microalbuminuria?: contra'. Kidney Int 2014;86:50-57.

15 R.J. MacIsaac, G. Jerums, M.E. Cooper: New insights into the significance of microalbuminuria. Curr Opin Nephrol Hypertens 2004;13:83-91. 


\section{Kidney \\ Blood Pressure Research}

16 Hogan SL, Vupputuri S, Guo X, Cai J, Colindres RE, Heiss G, Coresh J: Association of cigarette smoking with albuminuria in the United States: the third National Health and Nutrition Examination Survey. Ren Fail 2007;29:133-142.

17 Orth SR: Smoking - a renal risk factor. Nephron 2000;86:12-26.

-18 Morris PB, Ference BA, Jahangir E, Feldman DN, Ryan JJ, Bahrami H, El-Chami MF, Bhakta S, Winchester DE, Al-Mallah MH, Sanchez Shields M, Deedwania P, Mehta LS, Phan BA, Benowitz NL: Cardiovascular Effects of Exposure to Cigarette Smoke and Electronic Cigarettes: Clinical Perspectives From the Prevention of Cardiovascular Disease Section Leadership Council and Early Career Councils of the American College of Cardiology. J Am Coll Cardiol 2015;66:1378-1391.

19 Jarup L, Berglund M, Elinder CG, Nordberg G, Vahter M: Health effects of cadmium exposure-a review of the literature and a risk estimate. Scand J Work Environ Health 1998;24:1-51.

20 Satarug S, Moore MR: Adverse health effects of chronic exposure to low-level cadmium in foodstuffs and cigarette smoke. Environ Health Perspect 2004;112:1099-1103.

-21 Ritz E, Benck U, Franek E, Keller C, Seyfarth M, Clorius J: Effects of smoking on renal hemodynamics in healthy volunteers and in patients with glomerular disease. J Am Soc Nephrol 1998;9:1798-1804.

22 Bardoux P, Bruneval P, Heudes D, Bouby N, Bankir L: Diabetes-induced albuminuria: role of antidiuretic hormone as revealed by chronic V2 receptor antagonism in rats. Nephrol Dial Transplant 2003;18:17551763.

-23 Bardoux P, Bichet DG, Martin H, Gallois Y, Marre M, Arthus MF, Lonergan M, Ruel N, Bouby N, Bankir L: Vasopressin increases urinary albumin excretion in rats and humans: involvement of V2 receptors and the renin-angiotensin system. Nephrol Dial Transplant 2003;18:497-506.

24 Maity P, Biswas K, Roy S, Banerjee RK, Bandyopadhyay U: Smoking and the pathogenesis of gastroduodenal ulcer--recent mechanistic update. Mol Cell Biochem 2003;253:329-338.

25 Rowe JW, Kilgore A, Robertson GL: Evidence in man that cigarette smoking induces vasopressin release via an airway-specific mechanism. J Clin Endocrinol Metab 1980;51:170-172.

-26 Enhörning S, Bankir L, Bouby N, Struck J, Hedblad B, Persson M, Morgenthaler NG, Nilsson PM, Melander O: Copeptin, a marker of vasopressin, in abdominal obesity, diabetes and microalbuminuria: the prospective Malmö Diet and Cancer Study cardiovascular cohort. Int J Obes (Lond) 2013;37:598-603.

-27 van Gastel MD, Meijer E, Scheven LE, Struck J, Bakker SJ, Gansevoort RT: Modifiable factors associated with copeptin concentration: a general population cohort. Am J Kidney Dis 2015;65:719-727.

28 Orth SR, Ritz E: Adverse effect of smoking on renal function in the general population: are men at higher risk? Am J Kidney Dis 2002;40:864-866.

29 Helou R: Should we continue to use the Cockcroft-Gault formula? Nephron Clin Pract 2010;116:172-185.

-30 Szummer K, Evans M, Carrero JJ, Alehagen U, Dahlström U, Benson L, Lund LH: Comparison of the Chronic Kidney Disease Epidemiology Collaboration, the Modification of Diet in Renal Disease study and the Cockcroft-Gault equation in patients with heart failure. Open Heart 2017;4:e000568. 